\title{
Mass-imbalanced Fermi gases with spin-orbit coupling
}

\author{
M. Iskin ${ }^{1}$ and A. L. Subaş ${ }^{2}$ \\ ${ }^{1}$ Department of Physics, Koç University, Rumelifeneri Yolu, TR-34450 Sar1yer, Istanbul, Turkey \\ ${ }^{2}$ Department of Physics, Faculty of Science and Letters, Istanbul Technical University, TR-34469 Maslak, Istanbul, Turkey
}

(Received 12 July 2011; published 31 October 2011)

\begin{abstract}
We use the mean-field theory to analyze the ground-state phase diagrams of spin-orbit-coupled massimbalanced Fermi gases throughout the BCS-BEC evolution, including both the population-balanced and -imbalanced systems. Our calculations show that the competition between the mass and population imbalance and the Rashba-type spin-orbit coupling gives rise to very rich phase diagrams, involving normal, superfluid, and phase-separated regions. In addition, we find quantum phase transitions between the topologically trivial gapped superfluid and the nontrivial gapless superfluid phases, opening the way for the experimental observation of exotic phenomena with cold atom systems.
\end{abstract}

DOI: $10.1103 /$ PhysRevA.84.041610

PACS number(s): 03.75.Ss, 05.30.Fk, 03.75.Hh

Introduction. Following great successes with single-species (mass-balanced) two-component Fermi gases [1,2], there has been increasing experimental interest in realizing and studying two-species (mass-imbalanced) Fermi-Fermi mixtures over the past few years $[3,4]$. So far the most prominent candidate seems to be the ${ }^{6} \mathrm{Li}^{-}{ }^{40} \mathrm{~K}$ mixtures, for which experimental methods are currently being developed in several groups. For instance, recently ${ }^{6} \mathrm{Li}^{-}{ }^{40} \mathrm{~K}$ mixtures have been trapped and interspecies Feshbach resonances have been identified, opening a frontier in ultracold atom research to study exotic many-body phenomena. We also note that several other fermionic atoms, including ${ }^{171} \mathrm{Yb}$ [5] and ${ }^{87} \mathrm{Sr}[6]$, are also currently being investigated, allowing for future mixture experiments with various other species. Motivated partly by these experiments, and also due to the natural way of creating superfluidity with mismatched Fermi surfaces, there has also been increasing theoretical interest in understanding and studying two-species mixtures $[7,8]$.

In addition to these developments, following the recent realization of synthetic gauge fields with neutral bosonic atoms [9] and the more recent creation of spin-orbit-coupled (SOC) Bose-Einstein condensates (BECs) [10], it is now possible to create and study spin-orbit-coupled Fermi gases by making use of similar experimental methods [11]. Since this technique allows for the realization of topologically nontrivial states in atomic systems, with possibly broad interest for the physics community [12-14], there has been increasing theoretical interest in studying the effects of SOC on single-species two-component Fermi gases. For instance, it has been found for the population-balanced Fermi gases that the SOC increases the single-particle density of states, which in return favors the Cooper pairing so significantly that by increasing the SOC while the scattering length is held fixed eventually induces a BCS-BEC crossover even for a weakly interacting system when $a_{s} \rightarrow 0^{-}$[15-17]. The increased density of states also has important effects on the population-imbalanced Fermi gases $[18,19]$, for which we have recently found that the SOC and population imbalance are counteracting, and that this competition tends to stabilize the uniform superfluid phase against the phase separation. In addition, topological quantum phase transitions associated with the appearance of momentum space regions with zero quasiparticle energies have been found, the signatures of which could be observed in the momentum distribution $[18,19]$.

In this Rapid Communication, we extend our recent work [18] to the case of mass- and population-imbalanced Fermi gases, and study the effects of spin-orbit coupling on the ground-state phase diagrams across a Feshbach resonance, i.e., throughout the BCS-BEC evolution. Since it is not possible to have a spin-orbit field that converts different species of atoms into each other, it may not be possible to create spin-orbit-coupled mass-imbalanced systems with two-species mixtures. However, mass-imbalanced systems can be engineered with single-species two-component Fermi gases loaded into spin-dependent optical lattices in such a way that the components have different effective masses.

Some of our main results are shown in Fig. 1, and they are as follows. The competition between the mass and population imbalance and the SOC gives rise to very rich phase diagrams, involving normal, superfluid, and phase-separated regions, and quantum phase transitions between the topologically trivial gapped superfluid and the nontrivial gapless superfluid phases. In addition, in sharp contrast to the no-SOC case where only the gapless superfluid phase can support population imbalance, both the gapless and gapped superfluid phases can support population imbalance in the presence of a SOC for all mass ratios including the mass-balanced Fermi gases. Similarly, again in sharp contrast to the no-SOC case where only the gapped superfluid phase can support population balance, both the gapped and gapless superfluid phases can support population balance in the presence of a SOC when the mass difference becomes large enough.

Mean-field theory. We obtain these results within the selfconsistent mean-field approximation. In the absence of a SOC and at low temperatures, it is well established that the meanfield theory is sufficient to describe the physics of Fermi gases both in the BCS and the BEC limits, and that this theory also captures qualitatively the correct physics in the entire BCSBEC evolution $[1,20]$. Hoping that the mean-field formalism remains sufficient in the presence of a SOC, which is expected since the system is weakly interacting both in the BCS and molecular BEC limits, here we analyze the ground-state phase diagrams of the system as a function of SOC, scattering and 

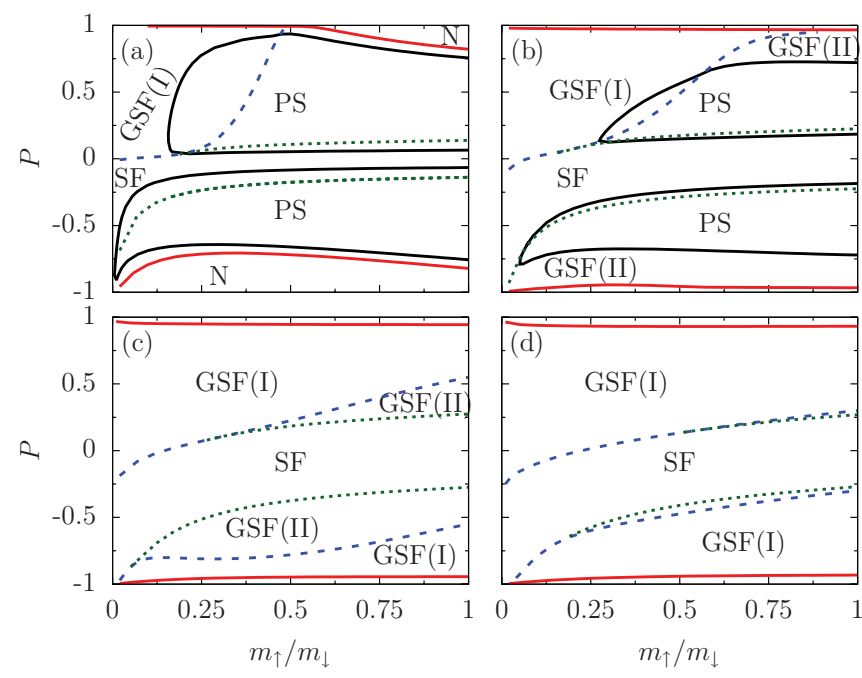

FIG. 1. (Color online) The ground-state phase diagrams are shown at unitarity, i.e., the scattering parameter is set to $1 /\left(k_{F} a_{s}\right)=0$, as a function of population imbalance $P=\left(N_{\uparrow}-N_{\downarrow}\right) / N$ and mass ratio $m_{\uparrow} / m_{\downarrow}$. Here the SOC parameter $\alpha m_{+} / k_{F}$ is set to 0.05 in (a), 0.15 in (b), 0.3 in (c), and 0.45 in (d), where $m_{+}$is twice the reduced mass of $\uparrow$ and $\downarrow$ fermions. We show normal (N), phase separation (PS), topologically trivial gapped superfluid (SF), and the topologically distinct gapless superfluid (GSF) phases.

population imbalance parameters, as well as the mass ratio of the fermions.

For this purpose, we use the mean-field Hamiltonian (in units of $\hbar=1=k_{B}$ )

$$
H=\frac{1}{2} \sum_{\mathbf{k}} \psi_{\mathbf{k}}^{\dagger}\left(\begin{array}{cccc}
\xi_{\mathbf{k}, \uparrow} & S_{\mathbf{k}} & 0 & \Delta \\
S_{\mathbf{k}}^{*} & \xi_{\mathbf{k}, \downarrow} & -\Delta & 0 \\
0 & -\Delta^{*} & -\xi_{\mathbf{k}, \uparrow} & S_{\mathbf{k}}^{*} \\
\Delta^{*} & 0 & S_{\mathbf{k}} & -\xi_{\mathbf{k}, \downarrow}
\end{array}\right) \psi_{\mathbf{k}}+C,
$$

where $\psi_{\mathbf{k}}^{\dagger}=\left[a_{\mathbf{k}, \uparrow}^{\dagger}, a_{\mathbf{k}, \downarrow}^{\dagger}, a_{-\mathbf{k}, \uparrow}, a_{-\mathbf{k}, \downarrow}\right]$ denotes the fermionic operators collectively, and $a_{\mathbf{k}, \sigma}^{\dagger}\left(a_{\mathbf{k}, \sigma}\right)$ creates (annihilates) a spin$\sigma$ fermion with momentum k. Here, $C=(1 / 2) \sum_{\mathbf{k}, \sigma} \xi_{\mathbf{k}, \sigma}+$ $|\Delta|^{2} / g$ is a constant, and $\xi_{\mathbf{k}, \sigma}=\epsilon_{\mathbf{k}, \sigma}-\mu_{\sigma}$ with $\epsilon_{\mathbf{k}, \sigma}=$ $k^{2} /\left(2 m_{\sigma}\right)$ the kinetic energy, $\mu_{\sigma}$ the chemical potential, and $k=\sqrt{k_{x}^{2}+k_{y}^{2}+k_{z}^{2}}$. In addition, $S_{\mathbf{k}}=\alpha\left(k_{y}-i k_{x}\right)$ with strength $\alpha \geqslant 0$ is the Rashba-type SOC [21], and $\Delta=$ $g\left\langle a_{\mathbf{k}, \uparrow} a_{-\mathbf{k}, \downarrow}\right\rangle$ is the mean-field order parameter, where $\langle\cdots\rangle$ is a thermal average and $g \geqslant 0$ is the strength of the attractive particle-particle interaction which is assumed to be local.

The corresponding mean-field thermodynamic potential can be written as

$$
\Omega=T \sum_{\mathbf{k}, s} \ln \left[\frac{1+\tanh \left(\frac{E_{\mathbf{k}, s}}{2 T}\right)}{2}\right]-\frac{1}{2} \sum_{\mathbf{k}, s} E_{\mathbf{k}, s}+C,
$$

where $T$ is the temperature, $s= \pm$, and $E_{\mathbf{k}, s}^{2}=\xi_{\mathbf{k},+}^{2}+\xi_{\mathbf{k},-}^{2}+$ $|\Delta|^{2}+\left|S_{\mathbf{k}}\right|^{2}+2 s A_{\mathbf{k}}$ gives the quasiparticle excitation spectrum [13,14]. Here, $A_{\mathbf{k}}=\sqrt{\xi_{\mathbf{k},-}^{2}\left(\xi_{\mathbf{k},+}^{2}+|\Delta|^{2}\right)+\left|S_{\mathbf{k}}\right|^{2} \xi_{\mathbf{k},+}^{2}}$ and $\xi_{\mathbf{k}, s}=\epsilon_{\mathbf{k}, s}-\mu_{s}$, where $\epsilon_{\mathbf{k}, s}=\left(\epsilon_{\mathbf{k}, \uparrow}+s \epsilon_{\mathbf{k}, \downarrow}\right) / 2=k^{2} /\left(2 m_{s}\right)$, $m_{s}=2 m_{\uparrow} m_{\downarrow} /\left(m_{\downarrow}+s m_{\uparrow}\right)$, and $\mu_{s}=\left(\mu_{\uparrow}+s \mu_{\downarrow}\right) / 2$. Following the usual procedure, i.e., $\partial \Omega / \partial|\Delta|=0$ for the order parameter and $N_{\uparrow}+s N_{\downarrow}=-\partial \Omega / \partial \mu_{s}$ for the number equations, we obtain the self-consistency equations [18]

$$
\begin{array}{r}
-\frac{m_{+} V}{4 \pi a_{s}}=\frac{1}{2} \sum_{\mathbf{k}, s} \frac{\partial E_{\mathbf{k}, s}}{\partial|\Delta|^{2}} \tanh \left(\frac{E_{\mathbf{k}, s}}{2 T}\right)-\sum_{\mathbf{k}} \frac{m_{+}}{k^{2}}, \\
N_{\uparrow} \pm N_{\downarrow}=\frac{1}{2} \sum_{\mathbf{k}, s}\left[\frac{1 \pm 1}{2}+\frac{\partial E_{\mathbf{k}, s}}{\partial \mu_{ \pm}} \tanh \left(\frac{E_{\mathbf{k}, s}}{2 T}\right)\right] .
\end{array}
$$

Here, we eliminated the theoretical parameter $g$ in favor of the experimentally relevant $s$-wave scattering length $a_{s}$ via the relation $1 / g=-m_{+} V /\left(4 \pi a_{s}\right)+\sum_{\mathbf{k}} 1 /\left(2 \epsilon_{\mathbf{k},+}\right)$, where $m_{+}$is twice the reduced mass of $\uparrow$ and $\downarrow$ fermions and $V$ is the volume. The derivatives of the quasiparticle energies are given by $\partial E_{\mathbf{k}, s} / \partial|\Delta|^{2}=\left(1+s \xi_{\mathbf{k},-}^{2} / A_{\mathbf{k}}\right) /\left(2 E_{\mathbf{k}, s}\right)$ for the order parameter, $\partial E_{\mathbf{k}, s} / \partial \mu_{+}=-\left[1+s\left(\xi_{\mathbf{k},-}^{2}+\left|S_{\mathbf{k}}\right|^{2}\right) / A_{\mathbf{k}}\right] \xi_{\mathbf{k},+} / E_{\mathbf{k}, s}$ for the average chemical potential, and $\partial E_{\mathbf{k}, s} / \partial \mu_{-}=-[1+$ $\left.s\left(\xi_{\mathbf{k},+}^{2}+|\Delta|^{2}\right) / A_{\mathbf{k}}\right] \xi_{\mathbf{k},-} / E_{\mathbf{k}, s}$ for the half of the chemical potential difference.

We checked the stability of the mean-field solutions for the uniform superfluid phase using the curvature criterion [7], which says that the curvature of $\Omega$ with respect to $|\Delta|$, i.e.,

$$
\begin{aligned}
\frac{\partial^{2} \Omega}{\partial|\Delta|^{2}}= & \frac{|\Delta|^{2}}{2} \sum_{\mathbf{k}, s}\left\{-\frac{1}{T}\left(\frac{\partial E_{\mathbf{k}, s}}{\partial|\Delta|^{2}}\right)^{2} \operatorname{sech}^{2}\left(\frac{E_{\mathbf{k}, s}}{2 T}\right)\right. \\
& \left.+\left[s \frac{\xi_{\mathbf{k},-}^{4}}{A_{\mathbf{k}}^{3}}+4\left(\frac{\partial E_{\mathbf{k}, s}}{\partial|\Delta|^{2}}\right)^{2}\right] \frac{\tanh \left(\frac{E_{\mathbf{k}, s}}{2 T}\right)}{2 E_{\mathbf{k}, s}}\right\},
\end{aligned}
$$

needs to be positive. When the curvature $\partial^{2} \Omega / \partial|\Delta|^{2}$ is negative, the uniform mean-field solution does not correspond to a minimum of $\Omega$, and a nonuniform superfluid phase, e.g. a phase separation, is favored. It is known that the curvature criterion correctly discards the unstable solutions, but the metastable solutions may still survive. This may cause only minor quantitative changes in the first-order phase transition boundaries as shown for the mass-balanced Fermi gases [19]. Having set up the mean-field formalism, we are now ready to discuss the competition between normal fluidity, uniform superfluidity, and phase separation across a Feshbach resonance.

Ground-state phase diagrams. There are typically three phases in our phase diagrams. While the normal (N) phase is characterized by $\Delta=0$, the uniform superfluid and nonuniform superfluid, e.g., phase separation (PS), are characterized by $\partial^{2} \Omega / \partial|\Delta|^{2}>0$ and $\partial^{2} \Omega / \partial|\Delta|^{2}<0$, respectively, when $\Delta \neq 0$. Furthermore, in addition to the topologically trivial gapped superfluid (SF) phase, the gapless superfluid (GSF) phase can also be distinguished by the momentum-space topology of its excitations. Depending on the number of zeros of $E_{\mathbf{k}, s}$ (zero energy points in $\mathbf{k}$ space), there are two topologically distinct GSF phases: GSF(I), where $E_{\mathbf{k}, s}$ has two, and GSF(II), where $E_{\mathbf{k}, s}$ has four zeros. For the Rashba-type SOC, the zeros occur when $k_{\perp}=0$ and at real $k_{z}$ momenta, $k_{z, s}^{2}=B_{+}+s \sqrt{B_{-}^{2}-4 m_{\uparrow} m_{\downarrow}|\Delta|^{2}}$, provided that $|\Delta|^{2}<\left|B_{-}\right|^{2} /\left(4 m_{\uparrow} m_{\downarrow}\right)$ for $B_{+} \geqslant 0$, and $|\Delta|^{2}<-\mu_{\uparrow} \mu_{\downarrow}$ for $B_{+}<0$. Here, $B_{s}=m_{\uparrow} \mu_{\uparrow}+s m_{\downarrow} \mu_{\downarrow}$. The topologically trivial SF phase corresponds to the case where both $E_{\mathbf{k},+}$ and $E_{\mathbf{k},-}$ have no zeros and are always gapped. From this analysis, it follows that the conditions $|\Delta|^{2}=\left|B_{-}\right|^{2} /\left(4 m_{\uparrow} m_{\downarrow}\right)$ 

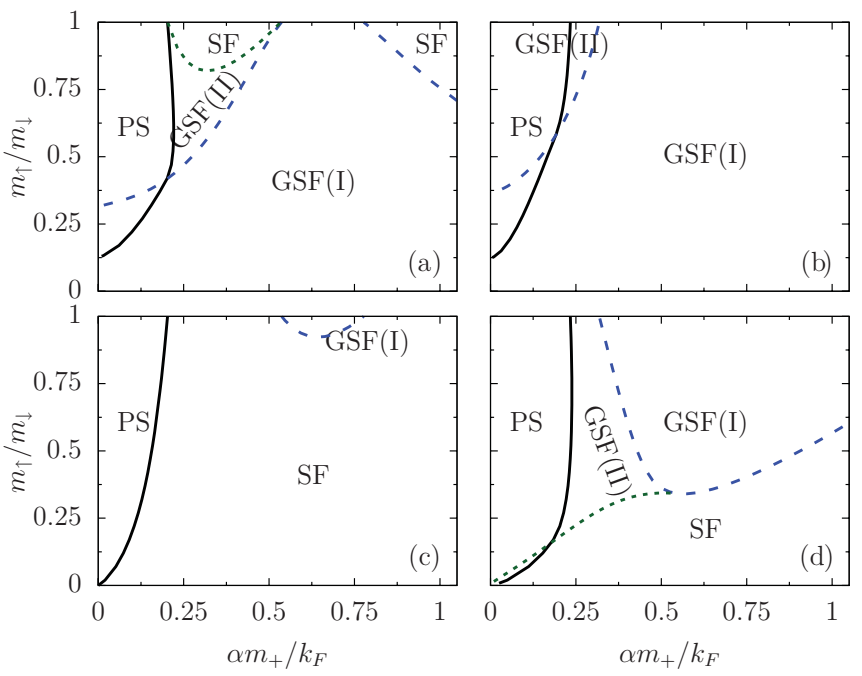

FIG. 2. (Color online) The ground-state phase diagrams are shown as a function of $m_{\uparrow} / m_{\downarrow}$ and $\alpha$ at $1 /\left(k_{F} a_{s}\right)=0$, where $P$ is set to 0.25 in (a), 0.5 in (b), -0.25 in (c), and -0.5 in (d). The labels are described both in Fig. 1 and in the text.

and $|\Delta|^{2}=-\mu_{\uparrow} \mu_{\downarrow}$ determine the phase boundaries between the SF, GSF(I), and GSF(II) regions, and that these three phases meet at a tricritical point determined by $B_{+}=0$ [18].

In our numerical calculations, we use an effective Fermi momentum $k_{F}$ and the corresponding Fermi energy $\epsilon_{F}=$ $k_{F}^{2} /\left(2 m_{+}\right)$as our length and energy scales, where $k_{F}$ is determined by fixing the total number of fermions $N=$ $N_{\uparrow}+N_{\downarrow}=k_{F}^{3} V /\left(3 \pi^{2}\right)$. In addition, we choose $\uparrow(\downarrow)$ to label lighter (heavier) fermions such that lighter (heavier) fermions are in excess when the population imbalance parameter $P=\left(N_{\uparrow}-N_{\downarrow}\right) / N$ is positive (negative). Therefore, since we choose $-1 \leqslant P \leqslant 1$, it is possible to span all possible mass-imbalanced Fermi gases by considering the mass ratios $0 \leqslant m_{\uparrow} / m_{\downarrow} \leqslant 1$.

(I) General phase diagrams. In Figs. 1 and 2, the groundstate phase diagrams are shown as a function of $P$ and $m_{\uparrow} / m_{\downarrow}$ for fixed $\alpha$ values, and as a function of $\alpha$ and $m_{\uparrow} / m_{\downarrow}$ for fixed $P$ values, respectively, at unitarity, i.e., $1 /\left(k_{F} a_{s}\right)=0$. The dashed (blue) and dotted (green) lines correspond to $|\Delta|^{2}=$ $-\mu_{\uparrow} \mu_{\downarrow}$ and $|\Delta|^{2}=\left|B_{-}\right|^{2} /\left(4 m_{\uparrow} m_{\downarrow}\right)$, respectively. Since our classification of distinct topological phases applies only to the uniform superfluid region, the dashed and dotted lines shown within the PS regions are solely for illustration purposes.

We find that the phase diagrams are symmetric around $P=0$ for mass-balanced systems, and that this symmetry is gradually broken as the mass difference increases. Due to this asymmetry, while the N, SF, and GSF(II) phases occupy much larger regions when heavier fermions are in excess, the PS and GSF(I) phases occupy larger regions when lighter fermions are in excess. It is clearly shown in these figures that increasing $\alpha$ gradually stabilizes the SF and GSF phases against the $\mathrm{N}$ and PS for all mass ratios, and mostly the SF and GSF(I) phases remain in the phase diagrams for very large $\alpha$. This is mainly a consequence of increased single-particle density of states due to the SOC as mentioned in the introduction.

In sharp contrast to the $\alpha=0$ case where only the gapless GSF phase can support population imbalance, one of the
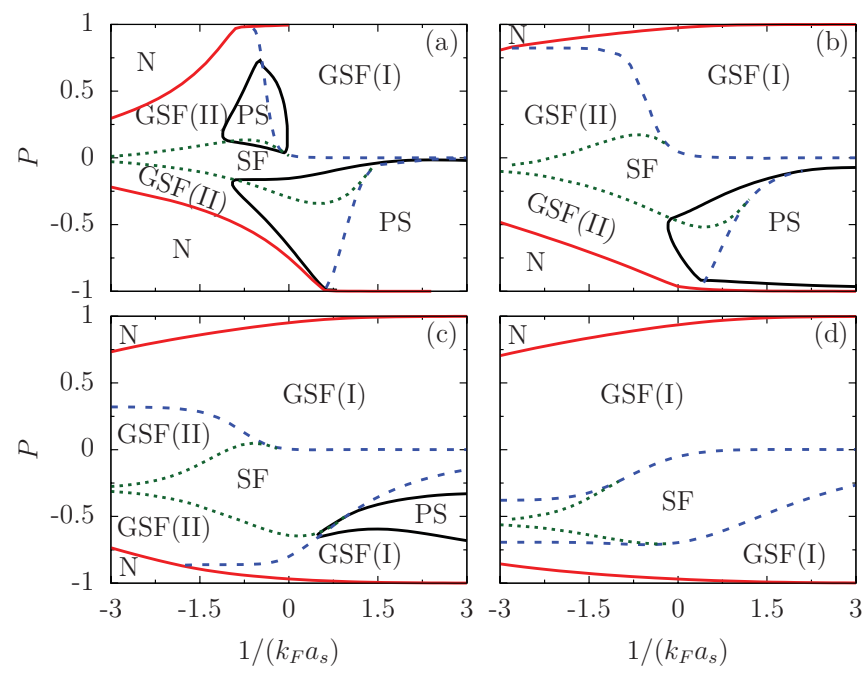

FIG. 3. (Color online) The ground-state phase diagrams of a Fermi gas with $m_{\uparrow}=0.15 m_{\downarrow}$ are shown as a function of $P$ and $1 /\left(k_{F} a_{s}\right)$, where $\alpha m_{+} / k_{F}$ is set to 0.05 in (a), 0.15 in (b), 0.3 in (c), and 0.45 in (d). The labels are described both in Fig. 1 and in the text.

intriguing effects of the SOC is that both the gapless GSF and gapped SF phases can support population imbalance when $\alpha \neq 0$. As shown in Figs. 1 and 2, this happens for all mass ratios, including the mass-balanced systems [18,19], but the gapped SF phase with population imbalance occupies a much larger region when the heavier fermions are in excess. Similarly, again in sharp contrast to the $\alpha=0$ case where only the gapped SF phase can support population balance, another intriguing effect of the SOC is that both the gapped SF and gapless GSF phases can support population balance for large enough mass differences when $\alpha \neq 0$. At unitarity, this occurs for all $\alpha$ values when $m_{\uparrow} \lesssim 0.5 m_{\downarrow}$, with the largest effect at $\alpha \approx 0.55 k_{F} / m_{+}$.

(II) Fermi gas with $m_{\uparrow}=0.15 m_{\downarrow}$. Having discussed the general structure of the phase diagrams as a function of
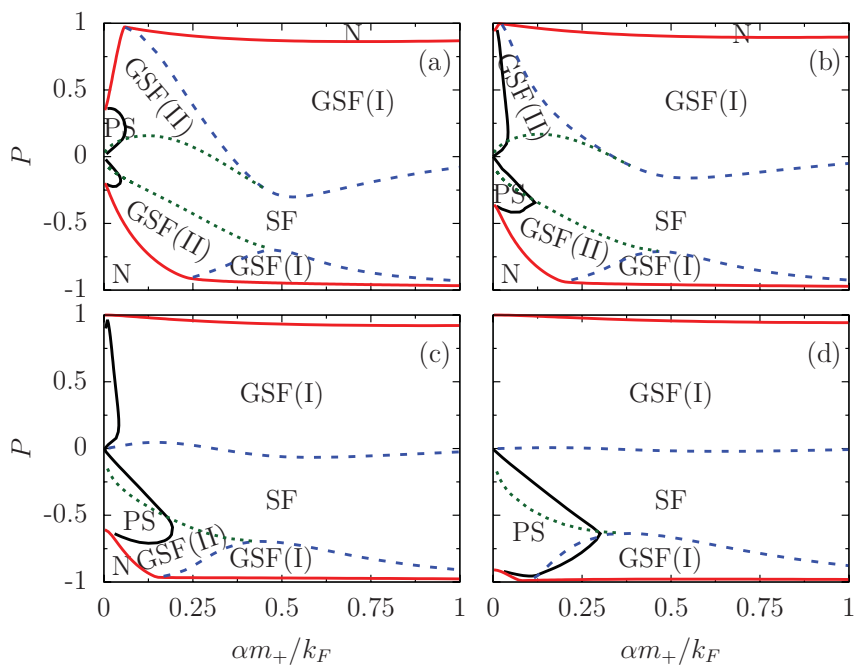

FIG. 4. (Color online) The ground-state phase diagrams of a Fermi gas with $m_{\uparrow}=0.15 m_{\downarrow}$ are shown as a function of $P$ and $\alpha$, where $1 /\left(k_{F} a_{s}\right)$ is set to -1 in (a), -0.5 in (b), 0 in (c), and 0.5 in (d). The labels are described both in Fig. 1 and in the text. 
mass ratio, here we fix $m_{\uparrow}=0.15 m_{\downarrow}$ and present a thorough discussion of the resultant phase diagrams in the entire BCSBEC evolution. This particular mass ratio corresponds to that of two-species ${ }^{6} \mathrm{Li}^{40} \mathrm{~K}$ mixtures which are currently being developed in several groups, making them the most prominent candidate for realizing a mass-imbalanced Fermi gas [3].

Our results are shown in Figs. 3 and 4, where the ground-state phase diagrams are shown as a function of $P$ and $1 /\left(k_{F} a_{s}\right)$ for fixed $\alpha$ values, and as a function of $P$ and $\alpha$ for fixed $1 /\left(k_{F} a_{s}\right)$ values, respectively. In addition to the general findings discussed above, we find that the gapped SF phase with population imbalance gradually disappears toward the BEC limit, giving way to the gapless GSF(I) phase. This is quite intuitive since, in this limit, the Fermi gas is expected to be well described by a much simpler Bose-Fermi description of paired fermions (molecular bosons) and unpaired (excess) fermions, consistent with the k-space topology of the GSF(I) phase [7]. In addition, we find that both the gapped SF and gapless GSF(II) phases occupy much larger regions for all $1 /\left(k_{F} a_{s}\right)$ values when heavier fermions are in excess. When $m_{\uparrow}=0.15 m_{\downarrow}$, we finally note that the gapless GSF(I) phase with population balance can be observed at unitarity for moderate $\alpha$ values through, for instance, measurements of the momentum distribution and single-particle spectral function [22].

Conclusions. To summarize, we analyzed the effects of SOC on the ground-state phase diagrams of mass-imbalanced Fermi gases throughout the BCS-BEC evolution. One way to engineer such a system is to load a two-component Fermi gas into a spin-dependent optical lattice such that the components have different effective masses. We showed that the competition between the mass and population imbalance and the SOC gives rise to very rich phase diagrams, involving normal, superfluid, and phase-separated regions, and quantum phase transitions between the topologically trivial gapped superfluid and the nontrivial gapless superfluid phases. According to our calculations, one of the intriguing effects of the SOC is that, in sharp contrast to the no-SOC case where only the gapless superfluid phase can support population imbalance, both the gapless and gapped superfluid phases can support population imbalance in the presence of a SOC. This occurs for all mass ratios including the mass-balanced systems. Another intriguing effect of the SOC is that, again in sharp contrast to the no-SOC case where only the gapped superfluid phase can support population balance, both the gapped and gapless superfluid phases can support population balance in the presence of a SOC when the mass difference becomes large enough.

Acknowledgments. This work is supported by the Marie Curie International Reintegration (Grant No. FP7-PEOPLEIRG-2010-268239), Scientific and Technological Research Council of Turkey (Career Grant No. TÜBITAK-3501110T839), and the Turkish Academy of Sciences (TÜBAGEBIP). We also thank Informatics Institute in Istanbul Technical University for computing resources (HPCL Grant No. 1005201003).
[1] Ultracold Fermi Gases, Proceedings of the International School of Physics "Enrico Fermi," Course CLXIV, Varenna, 2006, edited by M. Inguscio, W. Ketterle, and C. Salomon (IOS, Amsterdam, 2008).

[2] M. W. Zwierlein et al., Science 311, 492 (2006); G. B. Partridge et al., ibid. 311, 503 (2006); Y. Shin et al., Nature (London) 451, 689 (2008); N. Navon et al., Science 328, 729 (2010).

[3] A.-C. Voigt et al., Phys. Rev. Lett. 102, 020405 (2009); F. M. Spiegelhalder et al., ibid. 103, 223203 (2009); T. G. Tiecke et al., ibid. 104, 053202 (2010); L. Costa et al., ibid. 105, 123201 (2010); A. Ridinger et al., EPL 96, 33001 (2011); A. Trenkwalder et al., Phys. Rev. Lett. 106, 115304 (2011); V. V. Ivanov et al., ibid. 106, 153201 (2011).

[4] H. Hara, Y. Takasu, Y. Yamaoka, J. M. Doyle, and Y. Takahashi, Phys. Rev. Lett. 106, 205304 (2011).

[5] T. Fukuhara, Y. Takasu, M. Kumakura, and Y. Takahashi, Phys. Rev. Lett. 98, 030401 (2007); S. Taie et al., ibid. 105, 190401 (2010).

[6] M. K. Tey, S. Stellmer, R. Grimm, and F. Schreck, Phys. Rev. A 82, 011608(R) (2010).

[7] M. Iskin and C. A. R. Sá de Melo, Phys. Rev. Lett. 97, 100404 (2006); Phys. Rev. A 76, 013601 (2007); 77, 013625 (2008).

[8] S.-T. Wu, C.-H. Pao, and S.-K. Yip, Phys. Rev. B 74, 224504 (2006); G.-D. Lin, W. Yi, and L.-M. Duan, Phys. Rev. A 74, 031604(R) (2006); G. Orso, L. P. Pitaevskii, and S. Stringari, ibid. 77, 033611 (2008); G. J. Conduit, P. H. Conlon, and B. D.
Simons, ibid. 77, 053617 (2008); H. Guo, C. C. Chien, Q. Chen, Y. He, and K. Levin, ibid. 80, 011601 (2009); J. E. Baarsma, K. B. Gubbels, and H. T. C. Stoof, ibid. 82, 013624 (2010); R. B. Diener and M. Randeria, ibid. 81, 033608 (2010).

[9] Y.-J. Lin et al., Phys. Rev. Lett. 102, 130401 (2009).

[10] Y.-J. Lin et al., Nature (London) 471, 83 (2011).

[11] J. D. Sau, R. Sensarma, S. Powell, I. B. Spielman, and S. DasSarma, Phys. Rev. B 83, 140510 (2011).

[12] M. Sato, Y. Takahashi, and S. Fujimoto, Phys. Rev. Lett. 103, 020401 (2009).

[13] A. Kubasiak et al., Europhys. Lett. 92, 46004 (2010).

[14] M. Gong et al., e-print arXiv:1105.1796.

[15] J. P. Vyasanakere, S. Zhang, and V. B. Shenoy, Phys. Rev. B 84, 014512 (2011)

[16] Z.-Q. Yu and H. Zhai, e-print arXiv:1105.2250.

[17] Hui Hu et al., e-print arXiv:1105.2488.

[18] M. Iskin and A. L. Subaş1, Phys. Rev. Lett. 107, 050402 (2011).

[19] W. Yi and G.-C. Guo, Phys. Rev. A 84, 031608(R) (2011).

[20] C. Lobo, A. Recati, S. Giorgini, and S. Stringari, Phys. Rev. Lett. 97, 200403 (2006); S. Pilati and S. Giorgini, ibid. 100, 030401 (2008); I. Bausmerth, A. Recati, and S. Stringari, Phys. Rev. A 79, 043622 (2009); A. Gezerlis, S. Gandolfi, K. E. Schmidt, and J. Carlson, Phys. Rev. Lett. 103, 060403 (2009).

[21] L. P. Gor'kov and E. I. Rashba, Phys. Rev. Lett. 87, 037004 (2001).

[22] J. P. Gaebler et al., Nat. Phys. 6, 569 (2010). 